\title{
Fault Current Limiting Characteristics of Separated and Integrated Three-Phase Flux-Lock Type SFCLs
}

\author{
Sung-Hun Lim*
}

\begin{abstract}
The fault current limiting characteristics of the separated and the integrated three-phase flux-lock type superconducting fault current limiters (SFCLs) were analyzed. The three-phase fluxlock type SFCL consisted of three flux-lock reactors and three high- $\mathrm{T}_{C}$ superconducting (HTSC) elements. In the integrated three-phase flux-lock type SFCL, three flux-lock reactors are connected on the same iron core. On the other hand, three flux-lock reactors of the separated three-phase flux-lock type SFCL are connected on three separated iron cores. The integrated three-phase flux-lock type SFCL showed the different fault current limiting characteristics from the separated three-phase fluxlock type SFCL that the fault phase could affect the sound phase, which resulted in quench of the HTSC element in the sound phase. Through the computer simulation applying numerical analysis for its three-phase equivalent circuit, the fault current limiting characteristics of the separated and the integrated three-phase flux-lock type SFCLs according to the ground fault types were compared.
\end{abstract}

Keywords: Fault current limiting characteristics, Fault types, Separated and integrated three-phase flux-lock type SFCLs

\section{Introduction}

As the demand for the electrical power and the interconnection of electrical networks increased, the increase of the short-circuit current in power systems has accelerated. To reduce this short-circuit current, which has exceeded available cut-off ratings of existing circuit breakers, the various methods have been developed [1-2]. As one of methods, the superconducting fault current limiter (SFCL) has been expected to be the most promising power machine to limit the short-circuit current quickly and effectively without influence on a power system during normal operation. The SFCLs, which have been developed in many countries, can be largely classified into the quench type and the non-quench type SFCLs [3-6]. The flux-lock type SFCL belongs to the quench type SFCL from the point of view that the fault current can be limited by the occurrence of the superconductor's quench [7-8].

One advantage of the flux-lock type SFCL is that the initial operating current of the SFCL can be adjusted by the inductance ratio between coil 1 and coil 2 [7-8]. Another advantage is that the resistance of the high- $T_{C}$ superconducting (HTSC) element comprising the flux-lock type SFCL can be increased by the application of a magnetic field into the HTSC element without additional power supply and thus, the limiting current capacity of the flux-lock type SFCL can be increased [8].

\footnotetext{
* Department of Electrical Engineering, Soongsil University, 511, Sangdo-Dong, Dongjak-Gu, Seoul, 156-743, Korea

(e-mail: superlsh73@ssu.ac.kr)

Received 6 October, 2005 ; Accepted 4 April, 2006
}

Despite these merits, however, most researches for the flux-lock type SFCL have been mainly focused on the single-phase SFCL compared to other types of SFCLs. Therefore, the fault analyses for the three-phase system with the three-phase flux-lock type SFCL are required to apply the flux-lock type SFCL into a real power system.

In this paper, the operational characteristics of the integrated three-phase flux-lock type SFCL were analyzed and its fault current limiting characteristics were compared with the separated three-phase flux-lock type SFCL. Through the computer simulation using numerical analysis for each equivalent circuit, the fault current limiting characteristics for two three-phase flux-lock type SFCLs according to the threephase ground fault types were analyzed.

\section{Structure And Equivalent Circuit}

The structure of three-phase flux-lock type SFCL is shown in Fig. 1. Each single-phase circuit consists of two magnetically coupled coils connected in parallel and the HTSC element, which is connected in series with coil 2. As shown in Fig. 1(a), in case of the separated three-phase flux-lock type SFCL, each single-phase circuit is wound on each iron core and thus, magnetically separated from other phase circuits. On the other hand, in case of the integrated three-phase flux-lock type SFCL as seen in Fig. 1(b), three single-phase circuits are magnetically coupled through one iron core, which is expected to be more effective and more economical for the reduction of the weight and the volume of the three-phase SFCL using the iron core. 


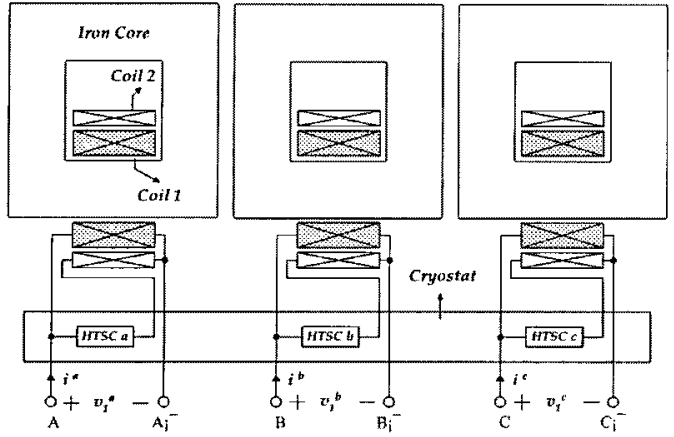

(a)

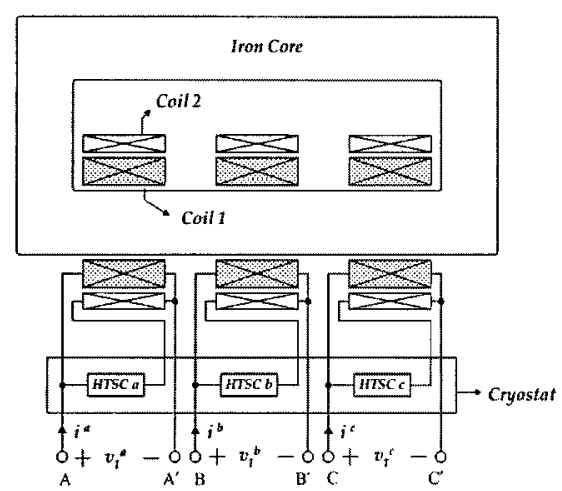

(b)

Fig. 1. Structure of the three-phase flux-lock type SFCL

(a) Separated three-phase flux-lock type SFCL

(b) Integrated three-phase flux-lock type SFCL

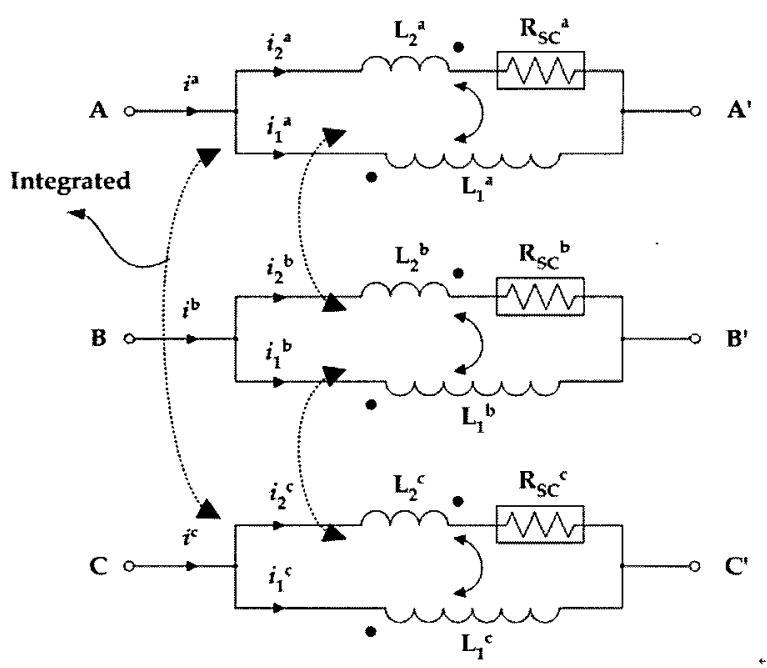

Fig. 2. Equivalent circuit of the three-phase flux-lock type SFCL

The equivalent circuit of three-phase flux-lock type SFCL is shown in Fig. 2. The arrow dotted lines, which describe the magnetic coupling between two phases, are included in the equivalent circuit of the integrated three- phase flux-lock type SFCL. In Fig. 2, superscript letters a, $\mathrm{b}$ and $\mathrm{c}$, which are used in current and voltage symbols, represent the indicator for each single-phase circuit, and subscript letters 1 and 2 are used to distinguish coil 1 from coil 2 in each single-phase circuit.

\section{Design Of Three-Phase Circuit With SFCL}

The three-phase circuit with SFCL for the analysis of the fault current limiting characteristics is shown in Fig. 3. The three-phase flux-lock type SFCL was installed between the power source and the transmission line. Design parameters of the three-phase circuit for computer simulation were listed in Table 1.

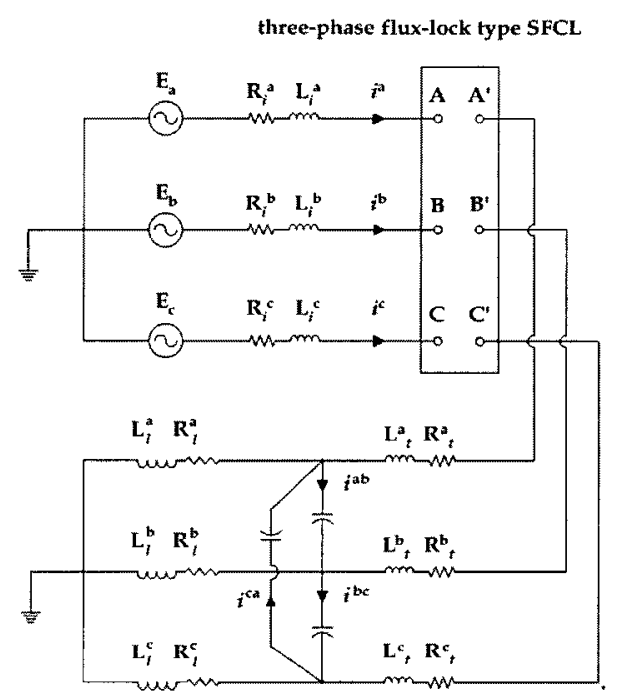

Fig. 3. Three-phase circuit with SFCL for computer simulation

Table 1. Parameter values of three-phase power system with SFCL for computer simulation

\begin{tabular}{lccc}
\hline Three-phase power system & Symbol & Value & Unit \\
\hline Phase voltage & $\mathrm{E}_{a}$ & 220 & $\mathrm{~V}_{\mathrm{rms}}$ \\
Resistance of power source & $\mathrm{R}_{i}$ & 0.01 & $\Omega$ \\
Inductance of power source & $\mathrm{L}_{i}$ & 0.1 & $\mathrm{mH}$ \\
Resistance of transmission line & $\mathrm{R}_{t}$ & 0.1 & $\Omega$ \\
Inductance of transmission line & $\mathrm{L}_{t}$ & 1 & $\mathrm{mH}$ \\
Load resistance & $\mathrm{R}_{l}$ & 50 & $\Omega$ \\
Load inductance & $\mathrm{L}_{l}$ & 1 & $\mathrm{mH}$ \\
Ground resistance & $\mathrm{R}_{n}$ & 1 & $\Omega$ \\
Ground inductance & $\mathrm{L}_{n}$ & 1 & $\mathrm{H}$ \\
\hline Integrated three-phase SFCL & $\mathrm{Symbol}$ & Value & Unit \\
\hline Turns in Coil 1 & $\mathrm{N}_{1}$ & 84 & Turns \\
Turns in Coil 2 & $\mathrm{N}_{2}$ & 21 & Turns \\
Coupling coefficient between & & 0.99 & \\
coils & & & \\
\hline HTSC element's resistance & $\mathrm{Symbol}$ & Value & Unit \\
\hline Time constant & $\mathrm{T}_{\mathrm{S}}$ & 0.028 & $\Omega$ \\
Convergent value & $\mathrm{R}_{\mathrm{SC}}$ & 20 & $\mathrm{~A}$ \\
Critical current & $\mathrm{I}_{\mathrm{C}}$ & 20 & \\
\hline
\end{tabular}


The resistance generation of the HTSC element comprising three-phase flux-lock type SFCL was reflected into the three-phase circuit with SFCL [9]. Fig. 4 shows the resistance curve of the HTSC element used in this simulation. For comparison, the measured resistance curve was also added in Fig. 4.

By applying the numerical analysis for the differential equations derived from Fig. 3 , the currents $\left(i_{1}{ }^{\mathrm{a}}, i_{2}{ }^{\mathrm{a}}, i_{1}{ }^{\mathrm{b}}, i_{2}{ }^{\mathrm{b}}\right.$, $i_{1}{ }^{\mathrm{c}}, i_{2}{ }^{\mathrm{c}}$ ) flowing into two coils in each single-phase were obtained.

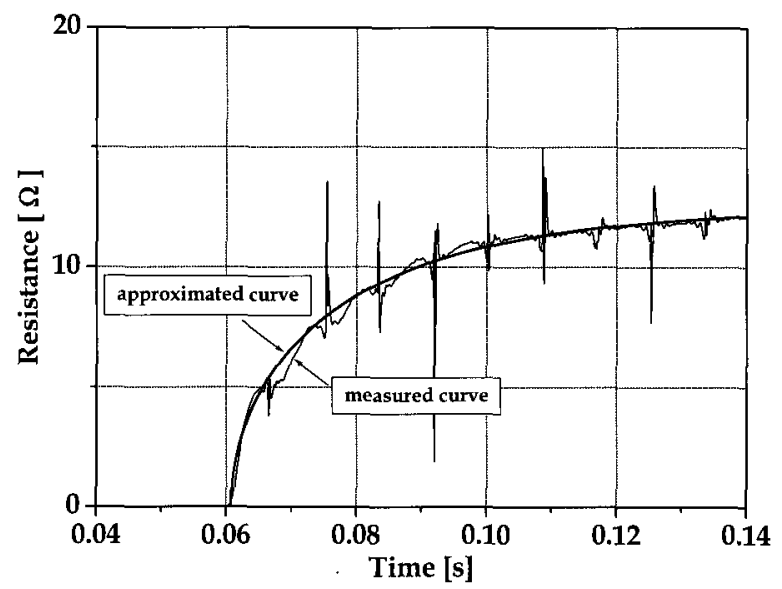

Fig. 4. Resistance curve of HTSC element used in this simulation

\section{Results And Discussions}

The fault current limiting characteristics of both the separated and the integrated three-phase flux-lock type SFCLs were compared for three-phase ground fault types. Fig. 5 shows the currents of the line, coil 1 and coil 2 in each single-phase in case that the single line-to-ground fault happens in a-phase of the separated three-phase fluxlock type SFCL. As seen Fig. 5, the currents corresponding to the fault phase only increased immediately after the fault occurrence and then, decreased by the operation of the single-phase flux-lock type SFCL installed in a fault phase. The fault current limiting characteristics of the integrated three-phase flux-lock type SFCL in case of the single lineto-ground fault were shown in Fig. 6. As seen in Fig. 6(a), the fault current of a phase was limited to $18 \%$ of the fault current of the short-circuit without the SFCL. Unlike separated three-phase flux-lock type SFCL, the quench in the HTSC elements of the $b$ and $c$ phase, which corresponded to the sound phases, occurred within 1/4 cycle after a fault happened. It was resulted from the magnetically coupled structure in one iron core. In addition, the amplitude and the phase of the currents passing through the HTSC elements in all the three phases became the same due to this magnetic coupling.
The fault current characteristics of the separated and the integrated three-phase flux-lock type SFCLs in case that the double line-to-ground fault occurs are illustrated in Figs. 7 and 8, respectively. As compared in case of the single line-to-ground fault, the fault current of the separated three-phase flux-lock type SFCL could be limited by the quench generation of HTSC elements correspond to the fault phase. On the other hand, in case of the integrated three-phase flux-lock type SFCL (Fig. 8), the quench in all the HTSC elements, irrespective of the fault phases, happened due to the magnetic coupling, which the current passing through the HTSC element in the sound phase exceeded its critical current by.

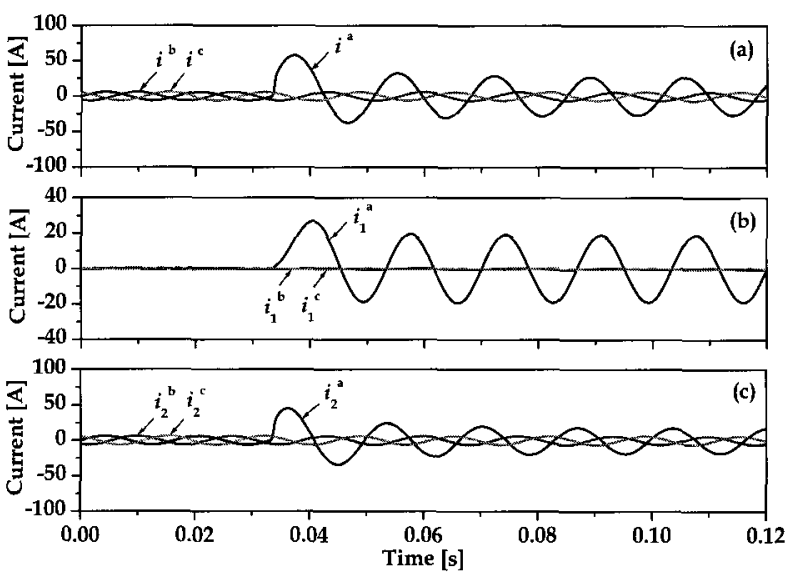

Fig. 5. Current waveforms in case of single line-to-ground fault in a separated three-phase flux-lock type SFCL.

(a) Line currents in each phase.

(b) Coil 1 currents in each phase.

(c) Coil 2 currents in each phase.
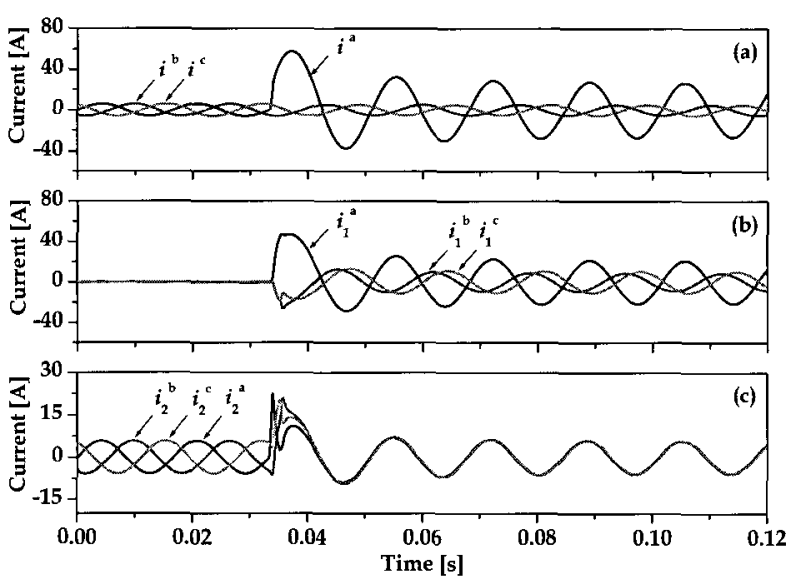

Fig. 6. Current waveforms in case of single line-to-ground fault in a integrated three-phase flux-lock type SFCL.

(a) Line currents in each phase.

(b) Coil 1 currents in each phase.

(c) Coil 2 currents in each phase. 
In case that the triple line-to-ground fault happened as shown in Figs. 9 and 10, the current all the HTSC elements comprising both the three-phase flux-lock type SFCLs exceeded the critical current of each HTSC element simultaneously and thus, the fault current could be limited by the resistance of HTSC element in each phase. In addition, it was observed that the symmetric characteristic of the triple line-to-ground fault, not the inner magnetic coupling of the iron core, accelerated the quench of all the HTSC elements comprising three-phase flux-lock type SFCL.

From the above fault analysis, we could observe that all the HTSC elements in the integrated three-phase flux-lock type SFCL were quenched by the magnetic coupling linked among three phases, irrespective of the ground fault types.
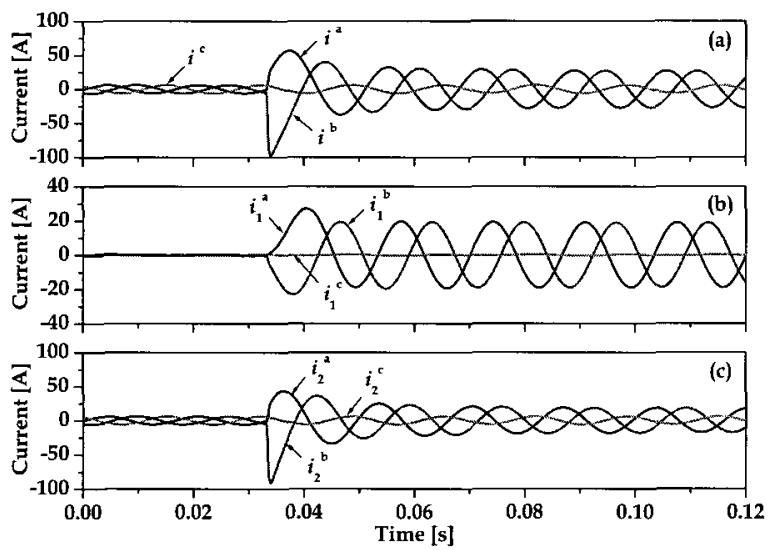

Fig. 7. Current waveforms in case of double line-to-ground fault in a separated three-phase flux-lock type SFCL.

(a) Line currents in each phase.

(b) Coil 1 currents in each phase.

(c) Coil 2 currents in each phase.

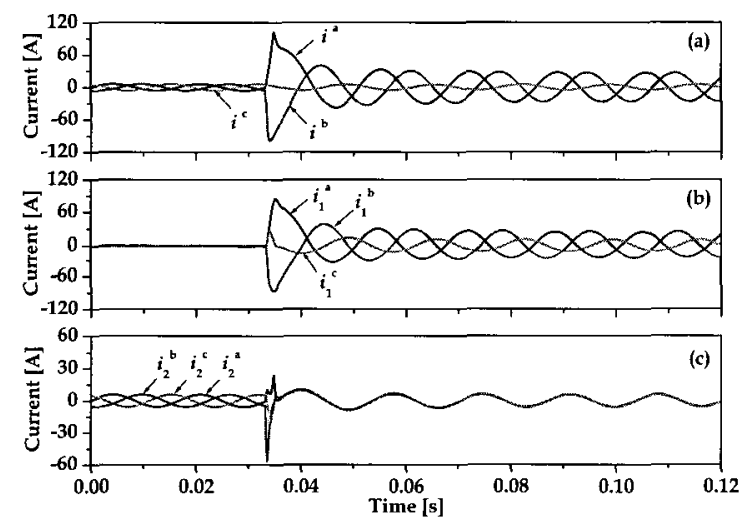

Fig. 8. Current waveforms in case of double line-to-ground fault in a integrated three-phase flux-lock type SFCL.

(a) Line currents in each phase.

(b) Coil 1 currents in each phase.

(c) Coil 2 currents in each phase.
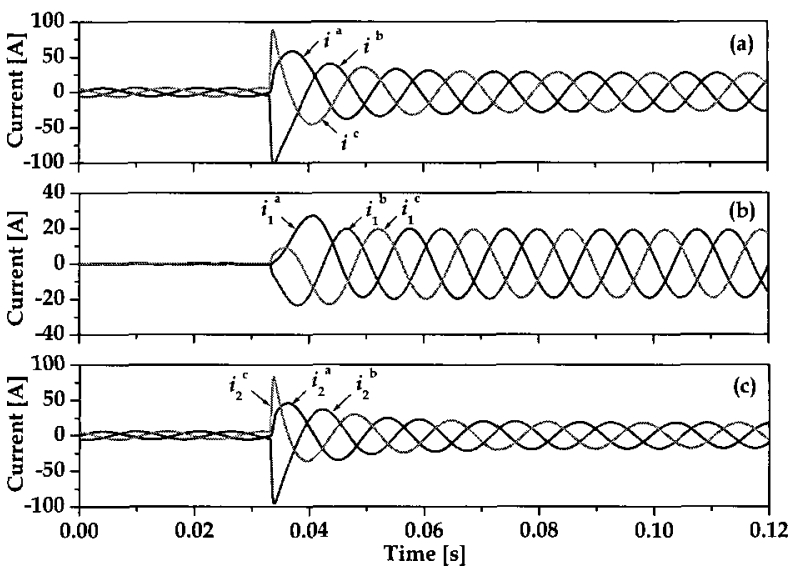

Fig. 9. Current waveforms in case of triple line-to-ground fault in a separated three-phase flux-lock type SFCL.

(a) Line currents in each phase.

(b) Coil 1 currents in each phase.

(c) Coil 2 currents in each phase.
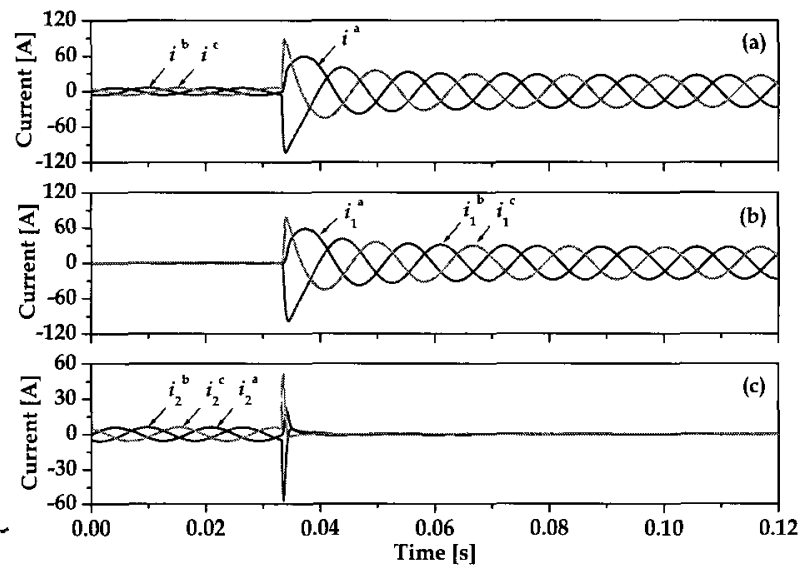

Fig. 10. Current waveforms in case of triple line-to-ground fault in a integrated three-phase flux-lock type SFCL.
(a) Line currents in each phase.
(b) Coil 1 currents in each phase.
(c) Coil 2 currents in each phase.

\section{Conclusions}

We compared the fault current limiting characteristics of the separated and the integrated three-phase flux-lock type SFCLs. The latter was a simple and economic structure compared to the former. It was found through the computer simulation applying numerical analysis for the equivalent circuit that in case of the integrated three-phase flux-lock type SFCL, the quenches in all the three HTSC elements happened irrespective of the ground fault types and that, on the other hand, the quench in the HTSC element corresponding to the fault phase happened in case of the separated three-phase flux-lock type SFCL. 


\section{Acknowledgement}

This work was supported by the Soongsil University Research Fund.

\section{References}

[1] L. Ye, L. Z. Lin, and K. P. Juengst, "Application studies of superconducting fault current limiters in electric power systems," IEEE Trans. Appl. Supercond., vol. 2, no. 9, pp. 900-903, March 2002.

[2] T. L. Mann, J. C. Zeigler, and T. R. Young, "Opportunities for superconductivity in the electric power industry," IEEE Trans. Appl. Supercond., vol. 7, no. 2, pp. 239-244, June 1997.

[3] B. Gromoll, G. Ries, W. Schmidt, H. P. Kraemer, B. Seebacher, B. Utz, R. Nies, and H. W. Newmuller, "Resistive fault current limiters with YBCO films 100kVA functional model," IEEE Trans. Appl. Supercond., vol. 9, no. 2, pp. 656-659, June 1999.

[4] H. Kado, and M. Ichikawa, "Performance of a high-Tc superconducting fault current limiter design of a $6.6 \mathrm{kV}$ magnetic shielding type superconducting fault current limiter," IEEE Trans. Appl. Supercond, vol. 7, no. 2, pp. 993-996, June 1997.

[5] M. Yamaguchi, S. Fukui, T. Satoh, Y. Kaburaki, and T. Horikawa, "Performance of DC reactor type fault current limiter using high temperature superconducting coil," IEEE Trans. Appl. Supercond, vol. 9 , no. 2, pp. 940-943, June 1999.

[6] T. Matsumura, T. Uchii, and Y. Yokomizu, "Development of flux-lock-type current limiter with high-Tc superconducting element," IEEE Trans. Appl. Supercond., vol. 7, no. 2, pp. 1001-1004, June 1997.

[7] S. H. Lim, H. S. Choi, and B. S. Han, "The fault current limiting characteristics of a flux-lock type high-Tc superconducting fault current limiter using series resonance," Cryogenics, vol. 44, pp. 249-254, April 2004.

[8] S. H. Lim, H. S. Choi, and B. S. Han, "Fault current limiting characteristics due to winding direction between coil 1 and coil 2 in a flux-lock type SFCL," Physica C, vol. 416, pp. 34-42, November 2004.

[9] S. H. Lim, S. R. Lee, H. S. Choi, and B. S. Han, "Analysis of Operational Characteristics of Flux-Lock Type SFCL with Combined with Power Compensator," IEEE Trans. Appl. Supercond., vol. 15, no. 2, pp. 2043-2046, June 2005.

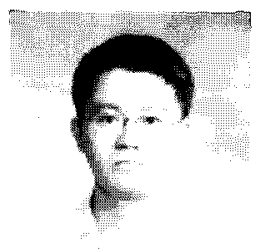

Sung-Hun Lim

He received his B.S., M.S. and Ph.D. degrees in Electrical Engineering from Chonbuk National University, Jeonju, South Korea in 1996, 1998 and 2003, respectively. He joined the faculty of Soong-Sil University, Seoul, Korea in 2006 where he is currently a Full-time Lecturer in the Department of Electrical Engineering. His current research interests include the application of superconductivity to power machines and power system. 